\title{
Ueber den Einfluss des Morphins auf die Fortbewegung des festen Magendarminhaltes hungernder Kaninchen.
}

\author{
Von
}

\author{
Stud. med. N. W. Krylow.
}

Die Untersuchungen Swirski' ${ }^{1}$ ) haben (ergeben, dass beim Kaninchen, welches, mit einem Maulkorbe versehen, hungert, nach Ablauf von $4 \times 24$ Stunden spätestens, im Magen kein Inhalt sich vorfindet, der von der vorher eingeführten Nahrung herrührt. Den Inhalt bildet meistens eine mit Haaren des Thieres vermischte schleimige, sauer reagirende Flüssigkeit, die durchschnittlich $0,3 \mathrm{~g}$, bei $100^{\circ} \mathrm{C}$. getrocknet, wiegt. Bei den Kaninchen jedoch, die ohne Maulkorb hungern, fand Swirski, dass nach Ablauf von $4-8 \times 24$ Stunden immer noch Inhalt vorhanden war, der aber auf verzehrten Koth zurückzuführen ist. Es betrug der bei $100^{\circ} \mathrm{C}$. getrocknete Mageninhalt bei diesen Thieren im Durchschnitte $3,5 \mathrm{~g}$.

Es musste nun von Interesse sein, zu untersuchen, wie sich die Fortbewegung des Magendarminhaltes der Kaninchen beim Hungern mit und ohne Maulkorb unter Anwendung von pharmakologischen Agentien, denen ein Einfluss auf den Magendarmcanal zukommt; verhalten würde. Mir fiel die Aufgabe zu, zunächst das Morphin in dieser Hinsicht zu prüfen.

Die Versuche wurden in folgender Weise ausgeführt. Nachdem die Kaninchen, wie Swirski angegeben, vorher mit Brot, Hafer und Klee reichlich gefüttert worden, wurden sie gewogen, mit einem gut sitzenden Maulkorb versehen und, nachdem ihnen Morph. hydrochl. entweder subcutan injicirt oder per os vermittelst Schlundsonde eingeführt worden, in einen Käfig gebracht. Die Wägung fand täglich um dieselbe Zeit statt. Koth und Harn wurden sorgfältig gesammelt; der erstere zunächst bei gewöhnlicher Temperatur, sodann nach

1) G. Swirski, Ueber das Verhalten des festen Magendarminhaltes bei absoluter Carenz der Kaninchen. Arch. f. exper. Path. u. Pharmakol. Bd. 48 S. 282.1902. 
Trocknung bei $100^{\circ} \mathrm{C}$. gewogen. Vom Harne wurde die Menge, das specifische Gewicht, die Reaction und der resp. Eiweissgehalt bestimmt.

Nachdem die Thiere so $3-5 \times 24$ Stunden gehungert hatten, wurden sie, falls sie nicht schon von selbst umgekommen waren, durch Entbluten getödtet und der Magendarmcanal genau nach der von Swirski ${ }^{1}$ ) angegebenen Art und Weise behandelt.

Der ganze Magendarmcanal wurde so in vier Abschnitte zerlegt: 1. Magen; 2. Dünndarm (vom Beginn des Duodenums bis zum Eintritt des Dünndarms in den Blinddarm am Beginne des Sacculus rotundus); 3. Blinddarm (vom Beginne des Sacculus rotundus bis zum Anfange des Colons); 4. Dickdarm (vom Beginne des Colons, kenntlich an den drei Längsstreifen, Taeniae coli, bis zum tiefsten von der Bauchhöhle aus erreichbaren Abschnitte des Rectums).

Zur Untersuchung mit Morph. hydrochl. kamen neun Kaninchen, von denen sieben dem Hunger mit Maulkorb, zwei dem Hunger ohne einen solchen unterworfen wurden. Ausser einem Kaninchen $(\boldsymbol{E})$, welchem das Morphin mit $\mathrm{H}_{2} \mathrm{O}$ vermittelst Schlundsonde direct in den Magen eingeführt wurde, erhielten alle übrigen das Morphin als subcutane Injection.

In der folgenden Tabelle gebe ich die Zahlen für die bei $100^{\circ} \mathrm{C}$. getrockneten Inhalte der einzelnen Abschnitte des Magendarmcanals der Kaninchen, die mit und ohne Maulkorb unter Morphin gehungert haben.

Tabelle I.

Hunger mit Maulkorb und Morphin.

\begin{tabular}{|c|c|c|c|c|c|c|c|c|}
\hline $\begin{array}{c}\text { Kanin- } \\
\text { chen }\end{array}$ & $\begin{array}{c}\text { Hunger- } \\
\text { dauer }\end{array}$ & $\begin{array}{l}\text { Körper- } \\
\text { gewicht }\end{array}$ & Magen & $\begin{array}{l}\text { Dünn- } \\
\text { darm }\end{array}$ & $\begin{array}{l}\text { Blind- } \\
\text { darm }\end{array}$ & $\begin{array}{l}\text { Dick- } \\
\text { darm }\end{array}$ & $\begin{array}{c}\text { Magen- } \\
\text { darm }\end{array}$ & $\begin{array}{c}\text { Morphin } \\
\text { in } \\
\text { Summa }\end{array}$ \\
\hline$A$ & & 1 & 2.0236 & 1.7927 & 50 & .1179 & 8,1592 & \multirow{7}{*}{$\begin{array}{r}0,12 \\
0,18 \\
+0,20^{2} \\
0,27 \\
+0,26 \\
\{\text { per os } \\
0,66 \\
+0,76\end{array}$} \\
\hline$B$ & $4 \times 24$ & 1500 & 2,8106 & 1,3523 & 2,9837 & 1,6141 & 8,7610 & \\
\hline$C$ & $3 \times 24$ & 1180 & 3,8337 & 1,4430 & 5,8364 & 2,0564 & 13,1695 & \\
\hline$D$ & $4 \times 24$ & 2130 & 7,1906 & 1,1923 & 9,6609 & 3,2187 & 21,2625 & \\
\hline$E$ & $4 \times 24$ & 1270 & 4,4724 & 0,7627 & 4,6249 & 3,4920 & 13,3520 & \\
\hline$F$ & $5 \times 24$ & 1345 & 3,2118 & 1,1 & 7,7 & 2,7 & 14 & \\
\hline$G$ & $4 \times 24$ & 1320 & 5,7805 & 1,4503 & 14,0987 & 0,8775 & 22,1670 & \\
\hline \multicolumn{3}{|l|}{$\begin{array}{l}\text { Mittel } \\
\text { Mageni }\end{array}$} & $\begin{array}{l}4,1890 \\
1.0000\end{array}$ & $\begin{array}{l}3108 \\
3129\end{array}$ & 16442 & $\begin{array}{l}2,0673 \\
0,4935\end{array}$ & 14,4551 & - \\
\hline
\end{tabular}

1) Swirski, l. c. S. 283.

2) Das Pluszeichen in dieser und Tabelle II zeigt an, dass das Kaninchen selbst verendet ist. 
Ueber den Einfluss des Morphins etc.

Hunger ohne Maulkorb mit Morphin.

\begin{tabular}{|c|c|c|c|c|c|c|c|c|}
\hline $\begin{array}{c}\text { Kanin- } \\
\text { chen }\end{array}$ & $\begin{array}{l}\text { Hunger- } \\
\text { dauer }\end{array}$ & $\begin{array}{l}\text { Körper- } \\
\text { gewicht }\end{array}$ & Magen & $\begin{array}{l}\text { Dünn } \\
\text { darm }\end{array}$ & $\begin{array}{l}\text { Blind- } \\
\text { darm }\end{array}$ & $\begin{array}{l}\text { Dick- } \\
\text { darm }\end{array}$ & $\begin{array}{c}\text { Magen- } \\
\text { darm }\end{array}$ & $\begin{array}{c}\text { Morphin } \\
\text { in } \\
\text { Summa }\end{array}$ \\
\hline$\frac{H}{I}$ & $\begin{array}{l}3 \times 24 \\
4 \times 24\end{array}$ & $\begin{array}{l}1202 \\
1295\end{array}$ & $\begin{array}{r}9,3123 \\
11,5802\end{array}$ & $\begin{array}{l}2,4500 \\
1,3886\end{array}$ & $\begin{array}{l}10,5161 \\
11,3200\end{array}$ & $\begin{array}{l}3,0849 \\
4,4773\end{array}$ & $\begin{array}{l}25,3633 \\
28,7661\end{array}$ & $\begin{array}{l}0,56 \\
0,30\end{array}$ \\
\hline $\begin{array}{l}\text { itte } \\
\text { age }\end{array}$ & & & $\begin{array}{r}10,4462 \\
1,0000\end{array}$ & $\begin{array}{l}1,9193 \\
0,1837\end{array}$ & $\begin{array}{r}10,9180 \\
1,1054\end{array}$ & $\begin{array}{l}3,7811 \\
0,3613\end{array}$ & $\underline{27,0647}$ & - \\
\hline
\end{tabular}

Bevor wir zu einer Besprechung der gefundenen Zahlen übergehen, ist es unerlässlich, die Kothmengen zu kennen, die von den mit und ohne Maulkorb hungernden Kaninchen unter der Morphinwirkung ausgesehieden wurden. Wir wissen aus den Untersuchungen S wirski's ${ }^{1}$ ), dass in den ersten 24 Stunden des Maulkorbhungers von den Kaninchen Kothmengen ausgeschieden werden, die meistens die Norm übersteigen, und zwar findet die Entleerung schon einige Stunden nach Application des Maulkorbes statt. Es musste nun von Interesse sein, zu beobachten, ob die genannte Erscheinung sich bei dem Maulkorbhunger mit Morphin wiederholt oder durch die Einwirkung des letzteren ausfällt. Es durfte das letztere angenommen werden, da das Morphin bekanntlich die Peristaltik herabsetzt.

Tabelle II.

Hunger mit Maulkorb und Morphin.

\begin{tabular}{|c|c|c|c|c|c|c|}
\hline $\begin{array}{c}\text { Kanin- } \\
\text { chen }\end{array}$ & $1 \times 24$ & $2 \times 24$ & $3 \times 24$ & $4 \times 24$ & $5 \times 24$ & Summa \\
\hline $\begin{array}{l}A \\
B \\
C \\
D \\
D \\
E \\
\boldsymbol{F} \\
G\end{array}$ & $\begin{array}{r}5,6500 \\
12,8900 \\
4,7000 \\
19,3700 \\
13,6742 \\
11,5140 \\
10,7000\end{array}$ & $\begin{array}{r}1,9977 \\
7,5450 \\
10,1610 \\
0,5163 \\
7,0120 \\
7,3000 \\
5,9573\end{array}$ & $\begin{array}{r}0,6870 \\
3,6850 \\
+4,3388 \\
4,3570 \\
0 \\
4,6007 \\
+3,1917\end{array}$ & $\begin{array}{r}0,4250 \\
0,7280 \\
0 \\
0,8447 \\
+1,0038 \\
1,5100 \\
0\end{array}$ & $\begin{array}{l}0,5650 \\
0 \\
0 \\
0 \\
0 \\
3,6100 \\
0\end{array}$ & $\begin{array}{r}9,3247 \\
24,8480 \\
19,1998 \\
25,0985 \\
21,6900 \\
28,5347 \\
19,8490\end{array}$ \\
\hline ittel . & 11,2154 & 5,7842 & 2,9800 & 0,6445 & 0,5964 & 21,2206 \\
\hline
\end{tabular}

Hunger obne Maulkorb mit Morphin.

\begin{tabular}{|c|c|c|c|c|c|c|}
\hline$\underset{I}{H}$ & $\begin{array}{l}0 \\
0,8450\end{array}$ & $\begin{array}{l}0 \\
0\end{array}$ & $\mid \begin{array}{c}+2,5950 \\
0\end{array}$ & $\begin{array}{l}0 \\
0\end{array}$ & $\mid \begin{array}{c}0 \\
+0,8230\end{array}$ & $\begin{array}{l}2,5350 \\
1,6680\end{array}$ \\
\hline
\end{tabular}

1) Swirski, l. c. S. 296. 
Wir sehen aus der vorstehenden Tabelle zunächst, dass die Mittelzahl für die Kothentleerung in den ersten 24 Stunden um 3,5 g die Mittelzahl $^{1}$ ) der täglichen Ausleerungen normaler Kaninchen übersteigt. Wollen wir uns jedoch über den Grad der Beeinflussung der Fortbewegung des Darminhaltes durch Morphin orientiren, so berechnen wir die Mittelzahl für die Kothentleerung der fünf mit Maulkorb hungernden Kaninchen aus der Arbeit Swirski's und bringen die von uns gefundene Zahl in Abzug:

$$
\begin{array}{r}
24,3834 \\
21,2206 \\
\hline 3,1628
\end{array}
$$

$3,1628 \mathrm{~g}$ Koth sind also von den mit Maulkorb und Morphin hungernden Kaninchen weniger ausgeschieden worden, was einer Minderausscheidung von $12 \%$ entspricht. Da beim Hunger mit Maulkorb vom vierten Tage ab rund $0,3 \mathrm{~g}$ im Magen als fester Inhalt vorhanden ist, bei unseren ebenso hungernden und ausserdem mit Morphin behandelten Kaninchen im Durchschnitte 4,2 g im Magen sich vorfinden, so weist das unzweifelhaft auf eine durch das genannte Alkaloid hervorgerufene Verlangsamung der Fortbewegung des Inhaltes im genannten Abschnitte des Nahrungsschlauches hin, da durch Verwendung eines Maulkorbes die Einführung des Kothes ausgeschlossen ist.

Der Dünndarm zeigt keine bedeutende Verlangsamung der Fortbewegung seines Inhaltes. Immerhin ist eine geringe Vermehrung des letzteren, um ca. 0,3 , bei den Maulkorb-Morphinkaninchen zu constatiren.

Der Blinddarm, das zweite grosse Reservoir des Darminhaltes, weist eine auffallendere Füllung auf als derjenige der nur mit einem Maulkorb hungernden Kaninchen und ebenso schliesslich der Dickdarm.

Wir constatiren somit in allen Theilen des Nahrungsschlauches der mit Maulkorb und Morphin hungernden Kaninchen eine stärkere Füllung als bei denen, die nur mit einem Maulkorb versehen, gehungert haben.

Von Swirski ist als die Grenze für das Verweilen des Mageninhaltes beim Maulkorbhunger $4 \times 24$ Stunden angenommen. In welchem Maasse aber für die einzelnen Tage bis zu dem genannten Termin der Magen der Maulkorbkaninchen sich entleert, ist bis jetzt durch speciell hierauf gerichtete Versuche noch nicht fest-

1) Sie beträgt 7,7 und ist berechnet aus dem Mittel für die tägliche Kothausscheidung normaler Kaninchen in der citirten Arbeit Swirski's S. 294 und in dieser Arbeit S. 293. 
gestellt worden. Es wäre das wichtig, um zu bestimmen, zu welcher Zeit ein die Peristaltik herabsetzendes Mittel seine Wirkung entfaltet hat. Daher war es mir sehr angenehm, über einen Versuch zu verfügen, der einen Einblick in die genannten Verbältnisse gestattet. Herr Privadocent Dr. S wirski überliess mir die Benutzung eines. Versuches, wo einem Kaninchen nach vorhergegangener entsprechender Fütterung ein Maulkorb für $1 \times 24$ Stunden aufgesetzt wurde. Nach Ablauf genannter Frist wurde es getödtet und wie bekannt behandelt.

Kaninchen von $1320 \mathrm{~g}$ Gewicht hungert $1 \times 24$ Stunden mit einem Maulkorbe versehen. Durch Entbluten getödtet.

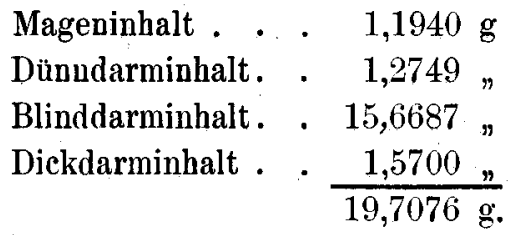

In 24 Stunden entleerter Koth auch bei $100^{\circ} \mathrm{C}$. getrocknet $=25,7550 \cdot$ Magendarminhalt und Koth $=45,4626 \mathrm{~g}$.

Dieser Versuch zeigt, dass im Verlaufe von 24 Stunden nach Aufsetzen eines Maulkorbes 25,7550 g Koth entleert wurden, welche Menge aus dem Coecum gekommen sein musste, da normaler Weise durchschnittlich 4,6 g im Dickdarme sich vorfinden. Es wäre also, die mittlere Füllung des Blinddarmes normaler Kaninchen mit $21 \mathrm{~g}$ angenommen, der ganze im letzteren befindliche Inhalt zusammen mit dem im Dickdarme befindlichen im Laufe der ersten 24 Stunden entleert worden. Dafür wären, entsprechend dem Verluste, aus dem Dünndarme und dem Magen, der ca. $16 \mathrm{~g}$ enthalten hätte, so viel fortgegangen, dașs eben im letzteren $1,1 \mathrm{~g}$, im Dünndarme 1,2 und im Blinddarme 15,6 Inhalt, bei $100^{\circ}$ C. getrocknet, nachblieben. Nehmen wir das Kaninchen $D$, das mit Maulkorb und Morphin hungerte, zum Vergleiche, da es $46,36 \mathrm{~g}$ in Summa für Koth und Magendarminhalt aufwies, eine Zahl, die ungefähr mit derjenigen des Kaninchens, das $1 \times 24$ Stunden hungerte, übereinstimmt. Da nun das Kaninchen $D$ im Verlaufe der ersten 24 Stunden des Hungers mit Morphin 19,37 g Koth ausschied, so musste diese Menge aus dem Dickdarm und Blinddarm stammen, und zwar würde letzterer bis auf $5 \mathrm{~g}$ ungefähr entleert worden sein, wenn wir annehmen, dass derselbe $\mathrm{zu}$ Beginn des Hungers mit $20 \mathrm{~g}$ Inbalt Trockengewicht gefüllt war. Es rückt nun unterdessen Inhalt aus dem 
Dünndarm und Magen nach, insgesammt ca. $14 \mathrm{~g}$, von denen $3 \mathrm{~g}$ auf den Dünndarm und 11 auf den Magen kommen. Von letzterer Menge würde 1,1 im Dünndarm verbleiben, während $13 \mathrm{~g}$ in den Blinddarm übergegangen wären. Wir fänden jetzt nach Ablauf von $1 \times 24$ Stunden im Magen $7 \mathrm{~g}$, im Dünndarme 1,1, im Blinddarm $18 \mathrm{~g}$. Da nun, wie wir aus der Tabelle I sehen, das Kaninchen $D$ nach Ablauf von $4 \times 24$ Stunden $7 \mathrm{~g}$ Inhalt im Magen und 1,1 im Dünndarme hatte, so ist anzunehmen, dass im Laufe der weiteren Hungertage aus dem Magen und dem Dünndarme kaum etwas weiter fortgerückt ist. Es wären also die genannten Theile auf dem Standpunkte stehen geblieben, welcher dem Abschlusse der ersten 24 Stunden des Hungers entsprach. Der Blinddarm hingegen hätte seine Thätigkeit noch fortgesetzt, da an zweiten Tage noch 0,5 , am dritten 4,3 und endlich am vierten Tage noch $0,8 \mathrm{~g}$ entleert wurden. Diese Entleerungen bewirkten es dann, dass der Blinddarm, der nach Ablauf der ersten 24 Stunden noch $18 \mathrm{~g}$ enthielt, durch successive Abgabe der soeben genannten Kothmengen allmählich auf 9,5 Inhalt angelangt ist, nachdem er noch $3,2 \mathrm{~g}$ zur Füllung des Dickdarms hat hergeben müssen.

Die Kaninchen, welche mit Morphin, aber ohne Maulkorb gehungert haben, zeigen im Magen eine stärkere, im Blinddarme hingegen eine schwächere Füllung als die Kaninchen, welche unter denselben Umständen, aber ohne Morphin hungern. Indem wir die nähere Besprechung dieser Verhältnisse auf Weiteres verschieben, möchten wir hier noch hervorheben, dass bei diesen Kaninchen unter der Morphinbehandlung sich die von Swirski gemachte Beobachtung, dass sie ihren Koth verzehren, wiederholte. Man konnte es dem Mageninhalte der Thiere sofort ansehen, dass er aus gefressenem Kothe bestand und zwar durchgängig. Es fand sich im Magen kein Inhalt, der auf den ursprünglich zu Beginn des Hungers bestehenden zurückzuführen war. Es wäre das sofort durch die grösseren Stücke der Hülsen von Getreidekörnern kenntlich gewesen. Der Inhalt bestand aus äusserst feinem dunkelbraunem Pulver, ähnlich feinem Schnupftabak. Der Kothkreislauf war also unter der Morphinbehandlung nicht unterbrochen worden. Während der Hungerzeit wurde von den Kaninchen durchschnittlich 2,1 $\mathrm{g}$ Koth ausgeschieden, also etwas mehr als von den ebenso, aber ohne Morphin hungernden Kaninchen, die $1,7 \mathrm{~g}$ aufwiesen.

Das Morphin. hydrochlor. wurde von mir in $2 \%$ iger Lösung 
in Einzelgaben, die zwischen $0,02-0,16 \mathrm{~g}$ schwankten, subcutan injicirt. Die Injectionen begannen sofort zu Beginn jedes Versuches und wurden im Laufe des Tages je nach Umständen wiederholt. Nur das Kaninchen $E$ erhielt täglich ein Mal das Morphin, 0,06 in $20 \mathrm{ccm}$ Wasser, vermittelst der Schlundsonde direct in den Magen. Die Injectionen riefen nur eine Somnolenz hervor, die sehr bald nach der Einführung des Mittels auftrat und je nach der Menge desselben längere oder kürzere Zeit andauerte. Nur in den letal endigenden Fällen wurde die Seitenlage einige Zeit vor dem Exitus eingenommen. Sobald dieser Zustand eintrat, wurde von einer Injection Abstand genommen. Von neun Kaninchen kamen fünf um, darunter auch beide ohne Maulkorb hungernde Kaninchen.

Erwähnt sei noch, dass die von den Maulkorb-Morphinkaninchen entleerten Harnmengen um mehr als das Dreifache diejenige der Maulkorbkaninchen überstiegen. Es schieden nämlich die letzteren im Mittel 52,7 cem Harn aus, die ersteren hingegen 163,7 ccm. Die Kothmengen der letzteren waren daher auch bedeutend weniger wasserreich als diejenigen der Maulkorbkaninchen.

Nachdem wir so mit den Folgen der Morphinbehandlung von Kaninchen, die mit und ohne Maulkorb hungerten, bekannt geworden sind, musste die Frage aufgeworfen werden, wie gut gefütterte Kaninchen sich in Bezug auf den Magendarmeanal bei Morphingebrauch verhalten würden.

Herr Privatdocent Dr. Swirski war so freundlich mir einen der von ihm beobachteten Fälle zu überlassen, aus dem man ersieht, dass das Morphin bei gut gefütterten, nicht hungernden Kaninchen keine die Peristaltik hemmende Wirkung hervorbringt.

Ein Kaninchen von $1698 \mathrm{~g}$ erbält als Futter nur Hafer und dazu Wasser. Im Verlaufe von sechs Tagen werden zuerst die täglich ausgeschiedenen Kothmengen gewogen und darauf im Verlaufe von weiteren sechs Tagen Morphin. hydrochlor. täglich ein Mal per os mit $35 \mathrm{ccm}$ Wasser eingeführt und zugleich das Gewicht der Kothmengen bestimmt.

17. November 1902: Kein Koth.

18.

$7,30 \mathrm{~g}$ Koth.

19.

$22,02, "$

$23,82 "$,

$15,65, "$

21 .

27,82,

1. Einführung von Morph. hydrochl. 0,06 g in $35 \mathrm{ccm}$ Wasser mit der Sonde.

$96,61 \mathrm{~g}$ Koth $=56,3907 \mathrm{~g}$ bei $100^{\circ} \mathrm{C}$. getrocknet. 


\begin{tabular}{|c|c|c|c|c|}
\hline & & Frisch gewogen & $\begin{array}{c}\text { Bei } 100^{\circ} \mathrm{C} . \\
\text { getrocknet }\end{array}$ & $\begin{array}{l}\text { Morph. } \\
\text { hydrochl }\end{array}$ \\
\hline \multicolumn{2}{|c|}{ 23. November: } & $15,27 \mathrm{~g}$ Koth & 9,8157 & 0,06 \\
\hline 24. & 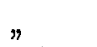 & $24,70 " n$ & 16,9747 & 0,06 \\
\hline 25. & $"$ & $15,40 "$ & 11,5415 & 0,06 \\
\hline 26. & $n$ & $22,57 "$ & 16,7758 & 0,12 \\
\hline 27. & $\eta$ & 10,32 & 5,7502 & 0,12 \\
\hline \multirow[t]{2}{*}{28.} & " & $7,50 \%$, & 4,3770 & 0,12 \\
\hline & & $95,76 \mathrm{~g} \mathrm{Koth}$ & 65,2349 & 0,60 \\
\hline
\end{tabular}

29. November. Dem Kaninchen wird ein Maulkorb aufgesetzt, nachdem vorher noch Morphin. hydrochlor., $0,12 \mathrm{~g}$ mit $50 \mathrm{ccm}$ Wasser, per Sonde in den Magen eingeführt worden. Die an diesem Tage vor Application des Maulkorbes ausgeschiedene Kothmenge betrug:

$$
\begin{array}{r}
18,27 \mathrm{~g} \text { Koth }=11,2134 \mathrm{~g} \\
\text { 30. November: } 21,30 ",=13,5012 " \\
\text { 1. December: } 13,76, "=8,9125,
\end{array}
$$

Der Maulkorb wird abgenommen, und das Kaninchen erhält von jetzt ab nur Kleeheu als Futter, dazu Wasser in gemessenen Quantitäten. Der Koth, der bis dahin eine gelbweissliche Farbe in Folge des Haferfutters hatte, wird unter der nun eingeschlagenen Fütterung allmählich braun. Der bis dahin saure Harn wird alkalisch. Der Harn enthielt 0,25-0,3\% Eiweiss nach Es bach.

6. December. Es hat sich jetzt beim Kaninchen ein colossaler Durst eingestellt. Die hingestellten $150 \mathrm{ccm}$ Wasser werden vom Thiere in einem Zuge ausgetrunken. Neu hingestellte $150 \mathrm{ccm}$ Wasser werden im Laufe des übrigen Tages verbraucht.

\section{December: $135,75, "=46,2893, "$}

$$
47,50 \mathrm{~g} \text { Koth }=15,3105 \mathrm{~g} \text { getrocknet. }
$$

Das Kaninchen ist sehr unruhig, sobald eine vorgesetzte ungenügende Menge Wassers verbraucht ist. Es erwartet mit Ungeduld neue Portionen. Harn $295 \mathrm{ccm}$ von stark alkalischer Reaction, 1007 spec. Gewicht; enthält kein Eiweiss und keinen Zucker. Das Gewicht des Kaninchens nimmt von Tag zu Tag ab, trotzdem es enorme Mengen von Kleeheu verzehrt. Der Durst besteht weiter; dementsprechend sind die entleerten Harnmengen colossal.

Nachdem das Thier immer schwächer geworden, liegt es schliesslich auf der Seite und verendet am 14. December. Der Magendarminhalt entsprach dem Maulkorbhunger von $1-2 \times 24$ Stunden, da das Kaninchen seinen Koth nicht mehr fressen konnte. 


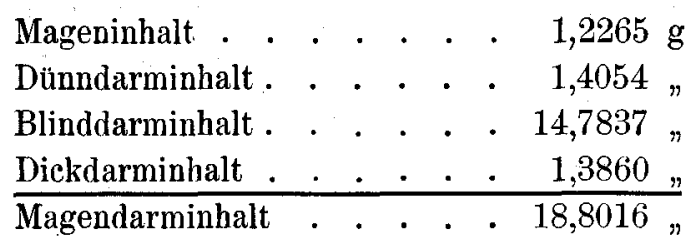

Aus diesem Versuche ersieht man, dass das Morphin, in Mengen von $0,06-0,12 \mathrm{~g}$ täglich per os eingeführt, bei gleichzeitiger reichlicher Fütterung nicht im Stande war, eine Behinderung der Kothentleerung herbeizuführen. Es stimmt das Gewicht des frisch entleerten Kothes für die der Morphinbehandlung vorhergehende Periode genau mit derjenigen der Morphinperiode überein. Ein Unterchied ist aber in den Trockengewichten: die während der Morphinbehandlung entleerten Fäces sind wasserärmer als die vorher ausgeschiedenen; letztere enthalten $42 \%$, erstere $32 \%$ Wasser. Es entspricht das dem, was wir bei den Maulkorb-Morphinkaninchen sahen, dass der Koth bei ihnen wasserärmer war als bei den nur mit Maulkorb hungernden. Wir sehen aber auch hieraus, dass die Eindickung der Fäces an sich nicht die Ursache für eine Behinderung der Fortbewegung des Magendarminhaltes sein kann, da hier beim normal gefütterten Kaninchen der geringere Wassergehalt der Fäces von keinen Folgen in Bezug auf Constipation war.

Dass die verabfolgten $0,7 \mathrm{~g}$ Morphin. hydrochlor. das Centralnervensystem stark geschädigt hatten, ist aus der schweren Erkrankung zu sehen, die unter den Symptomen des Diabetes insipidus zum Tode führte. Die Fütterungsweise des Thieres ist hierbei nicht im Spiele gewesen, da ganz gleich gefütterte Thiere durchaus nicht ähnliche Krankheitssymptome aufweisen.

Stellen wir die in unserer Arbeit für die einzelnen besprochenen Hungergruppen mit Morphin gefundenen Zahlen mit den normalen und den zwei Hungerformen ohne Morphin aus der schon citirten Arbeit von Swirski, den Mageninhalt $=1$ gesetzt, in einer Tabelle zusammen, so ergibt sich das auf S. 296 folgende Resultat:

Aus der Tabelle III ist ersichtlich, dass die ohne Maulkorb hungernden Morphinkaninchen den normalen am nächsten stehen und darauf die erste Gruppe der Maulkorb-Morphinkaninchen folgt, gebildet aus den Kaninchen $A-E$ (incl.) der Tabelle I, die mit $0,12-0,26 \mathrm{~g}$ Morphin. hydrochlor. behandelt worden. Die mit grossen Dosen des Alkaloides behandelten weichen schon stark ab von dem normalen, durch den mehr als das Doppelte des Mageninhaltes be- 
tragenden Blinddarminhalt. Von den normalen Kaninchen entfernen sich die ohne Morphin hungernden Kaninehen am meisten.

Tabelle III.

\begin{tabular}{c|c|c|c|c|c|c}
\hline \hline $\begin{array}{c}\text { Inhalt } \\
\text { vom }\end{array}$ & $\begin{array}{c}\text { Normale } \\
\text { Kaninchen }\end{array}$ & $\begin{array}{c}\text { Hunger } \\
\text { ohne } \\
\text { Maulkorb } \\
\text { mit } \\
\text { Morphin }\end{array}$ & $\begin{array}{c}\text { Maulkorb- } \\
\text { hunger } \\
\text { mit } \\
\text { Morphin } \\
0,14-0,27\end{array}$ & $\begin{array}{c}\text { Maulkorb- } \\
\text { hunger } \\
\text { mit } \\
\text { Morphin } \\
0,66-0,76\end{array}$ & $\begin{array}{c}\text { Ohne } \\
\text { Maulkorb } \\
\text { hungernde }\end{array}$ & Maulkorb- \\
kaninchen \\
\hline Magen. . . & 1,0000 & $\mathbf{1 , 0 0 0 0}$ & 1,0000 & 1,0000 & 1,0000 & 1,0000 \\
Dünndarm : & 0,1837 & 0,1731 & 0,3234 & 0,2928 & 0,2960 & 3,4764 \\
Blinddarm : & $\mathbf{1 , 0 2 4 3}$ & 1,1054 & 1,3015 & 2,4336 & 4,1642 & 12,9643 \\
Dickdarm . & 0,2233 & $\mathbf{0 , 3 6 1 3}$ & 0,5684 & 0,3305 & $\mathbf{1 , 0 8 3 1}$ & 2,2619 \\
\end{tabular}

Um die während der Morphinbehandlung im Darmeanale sich abspielenden Vorgänge einigermaassen $z u$ übersehen, ist es nöthig, die Summe aus den Darminhaltsgewichten und dem Koth der hungernden Morphinkaninchen mit derjenigen der ohne Morphin hungernden Kaninchen zu vergleichen.

\begin{tabular}{|c|c|c|c|}
\hline & $\begin{array}{c}\text { Magendarm- } \\
\text { inhalt }\end{array}$ & Koth & Summa \\
\hline $\begin{array}{l}\text { Mit Maulkorb hungernde Kaninchen } \\
\text { Mit Maulkorb a. Morphin hungernde }\end{array}$ & 5,6944 & 24,3834 & 30,0778 \\
\hline $\begin{array}{l}\text { Kaninchen } \\
\text { Ohne Maulkorb hungernde Kaninchen } \\
\text { Ohne Maulkorb mit Morphin hungernde }\end{array}$ & $\begin{array}{l}14,4551 \\
23,1090\end{array}$ & $\begin{array}{r}21,2206 \\
1,7131\end{array}$ & $\begin{array}{l}35,6757 \\
24,8222\end{array}$ \\
\hline Kaninchen . . . . . . & 27,0647 & 2,1015 & 29,1662 \\
\hline
\end{tabular}

Aus der Tabelle ersieht man, dass der Magendarminhalt bei beiden Arten des Hungerns unter der Morphinbehandlung an Gewicht zunimmt. Diese Gewichtszunahme kommt nun zu Stande nicht allein desswegen, weil weniger ausgeschieden wird, sondern auch offenbar desswegen, weil der Inhalt selbst zunimmt. Die mit Morphin hungernden Maulkorbkaninchen scheiden wobl $3,1628 \mathrm{~g}$ Koth weniger aus, aber im Magendarmeanal sind nach Abzug der genannten Zahl noch immer $5,5979 \mathrm{~g}$ mehr als bei den ohne Morphin hungernden Maulkorbkaninchen. Bei den ohne Maulkorb hungernden Kaninchen ist dieser Unterschied ebenfalls sehr deutlich. Bei den mit Morphin hungernden Kaninchen ist im Magendarmeanal $3,9 \mathrm{~g}$ Inhalt mehr vorhanden als bei den ohne Morphin hungernden. Wir haben es also bei den Morphinkaninchen ausser mit dem zurückgehaltenen Darminhalte noch mit dem sich neu bildenden zu thun. Es ist wohl sehr wahrscheinlich, dass im Magen, dem Orte, wo nach 
der Annahme von Marmé und Leineweber ${ }^{1}$ ) die Ausscheidung des Morphins erfolgt, auch schon ein Theil der aus dem Organismus sich abscheidenden Stoffwechselproducte ausgeschieden wird. Zum anderen Theile wird dann der weitere Darmcanal dafür verantwortlich zu machen sein. Dass aber im Magen bei den mit Maulkorb hungernden Morphinkaninchen noch ein Theil von dem vor dem Versuche eingeführten Futter nachzuweisen war, ist ohne Zweifel. Wie viel aber von dem gefundenen Inhalte auf vorher Eingeführtes und unter der Morphinbehandlung speciell Hinzugekommenes zurückzuführen war, ist von uns nicht weiter verfolgt worden. Wir nehmen an, dass das Plus auf den ganzen Mageudarmcanal gleichmässig vertheilt ist, um die Verhältnisse zu besprechen, unter denen die Vertheilung des Inhaltes auf den Nahrungsschlauch erfolgt ist. Hinweisen möchten wir nur noch auf den Umstand, dass die bei $100^{\circ} \mathrm{C}$. getrockneten einzelnen Darmabschnitte der Morphinkaninchen durchschnittlich leichter waren als die entsprechenden Theile bei den ohne Morphin hungernden Kaninchen. Ebenso war der Gewichtsuntersehied der genannten Theile bei den Morphinkaninchen unter einander ein geringerer als bei den beiden Hungerarten ohne Morphin unter einander.

Um die Wirkung des Morphins auf den Gastrointestinaltract der Kaninchen, wie wir sie in dieser Arbeit kennen gelernt haben, an der Hand der bekannten experimentellen Forschungen auf diesem Gebiete zu prüfen, erlauben wir uns in Kurzem auf die einschlägige Literatur einzugehen.

Die seiner Zeit von Nothnagel ${ }^{2}$ ) angenommene Erklärung der Morphinwirkung auf den Darm, dass es sich hauptsächlich um eine Reizung des Splanchnicuscentrums bei kleinen Morphindosen, bei grösseren hingegen um eine Lähmung des genannten Centrums handele, ist durch die nachfolgenden Arbeiten von $\mathrm{Pal}^{8}$ ) und $\mathrm{Berg-}$ g r ü $\mathrm{n}$ bestätigt worden.

Die Arbeiten von $\mathrm{Pohl}^{4}$ ) und $\mathrm{Jacobj}^{5}$ ) haben jedoch die An-

1) Marmé, Deutsche med. Wochenschr. $1883 \mathrm{Nr}$. 14.

2) Nothnagel, Beiträge zur Physiologie und Pathologie des Darmes S. 62. Berlin 1884.

3) Pal und Berggrün, Arbeiten aus den Institut für allgem. u. exper. Pathologie zu Wien. 1890.

4) Pohl, Ueber Darmbewegungen und ihre Beeinflussung durch Gifte. Arch. f. exper. Pathol, und Pharmakol. Bd. 34 S. 99. 1894.

5) J a cobj, Beiträge zur physiol. u. pharmakol. Kenntniss der Darmbewegungen. Arch. f. exper. Pathol. und Parmakol. Bd. 29 S. 210. 1892.

E. Pflüger, Arehiv für Physiologie. Bd. 102. 
gaben Nothnagel's, betreffend die centrale Wirkung des Morphins, nicht bestätigen können. Jacobj fand bei intravenöser Application von $0,08 \mathrm{~g}$ Morphin. hydrochlor. bei Kaninchen wohl eine geringe Abschwächung des Splanchnicustonus, aber keine wirkliche Lähmung des Centrums, denn nach Entfernung der Nebennieren erzeugte ClNa-Reizung eine ausgedehntere peristaltische Bewegung, wie auch Reizung des Vagus eine stärkere Peristaltik der Därme hervorrief. Diese Beobachtung stimmt mit der Mehrzahl der von Pohl an Kaninchen angestellten Versuche überein. Anders war aber das Resultat, wenn Jacobj in eine Darmschlinge 0,02 $\mathrm{g}$ Morphin. hydrochlor. injicirte. Vagusreizung brachte zuerst in der Schlinge, in die injicirt wurde, keine Bewegung hervor. 7 Minuten darauf konnte durch die Reizung der Vagi auch an dem gesammten Darme keine Bewegung mehr hervorgerufen werden. Opiumtinctur gab unter diesen Bedingungen ganz dasselbe Resultat. Jacobj kommt zum Schlusse, dass grössere Morphingaben den Hemmungstonus, der von den Nebennieren ausgeht oder vermittelt wird, nicht wesentlich herabsetzen, und dass es sich bei der innerlichen Anwendung des Morphins oder Opiums als Beruhigungmittel auf den Darm um eine locale Wirkung des Alkaloides auf in der Darmwand gelegene Apparate handelt. Reize, die sonst Bewegungen auszulösen im Stande sind, werden wirkungslos.

Eine vermittelnde Stellung nehmen Spitzer ${ }^{1}$ ) und v. Vámossy ${ }^{2}$ ) ein. Spitzer nimmt an, dass das Opium resp. Morphin durch Erregung centraler Hemmungen zum grösseren Theile, zum kleineren durch Herabsetzung der eigenen Empfindlichkeit des Darmes die Schwächung der Peristaltik erzeugt. Für die centrale Reizung sprach der Umstand, dass ein grösserer Unterschied im Verhalten der morphinisirten Thiere vor und nach Durchschneidung der Splanchnici vorhauden war als bei den Thieren, denen der Splanchnicus durchschnitten war, vor und nach der Morphinisirung. v. Vámossy hebt den Unterschied zwischen der intravenösen und der localen Application des Opiums rèsp. Morphins hervor. Bei intravenöser Einführung käme es zu einer Herabsetzung der Peri-

1) Spitzer, Experimental-Untersuchungen über die Darmwirkung des Opiums und Morphins. Virehow's Archiv Bd. 123 S. 622. 1891.

2) v. Vámossy, Zur Wirkung der Opium-Alkaloide auf die Darmbewegungen. Deutsche med. Wochenschr. 1897 p. 457. 
staltik durch Betäubung des Centrums, indem durch den Vagus centripetal geleitete Erregung im Gehirne verhindert wird, auf centrifugale darmbewegende Bahnen übertragen zu werden. Bei der Injection in den Darm konnte v. Vámossy die locale Beeinflussung der Darmganglien, wie $\mathrm{Pohl}$ und $\mathrm{Jacobj}$ sie fanden, constatiren.

Schmiedeberg ${ }^{1}$ ) erklärt die Herabsetzung der Peristaltik durch Morphin bei Durchfällen mit der Annahme, dass gewisse Nervenelemente in der Darmwand existiren, welche die vom Darmlumen her zu ihnen gelangenden Reize auf die ebenfalls in der Darmwand gelegenen motorischen, die Darmbewegungen vermittelnden nervösen Centra übertragen, und dass die Erregbarkeit dieser Nerven durch das Morphin vermindert wird. Eine Lähmung der motorischen Ganglien und der Muskeln ist bei der Morphinwirkung nach Schmiedeberg ausgeschlossen.

Nach dieser kurzen literarischen Umschau scheint es zunächst, als wenn ein Unterschied zwischen dem intravenös resp. subcutan eingeführten und dem per os in den Magen verabreichten Morphin zu machen wäre. Während das per os eingeführte durch Lähmung resp. Abstumpfung sensibler Apparate den Darm weniger erregbar mache für Reize, die ihn treffen, entfalte das intravenös oder subcutan eingeführte Morphin am Darme, selbst in grossen Dosen, fast gar keine Wirkung.

Für uns ist von Interesse, dass Ja cobj den Darm von Hungerkaninchen auf den Vagusreiz bei $120 \mathrm{~mm}$ R.-A. nicht reagiren sah, wohl aber nach Entfernung der Nebennieren. Da nun bei normal gefütterten Kaninchen Reizung des Vagus bei derselben Stromstärke kräftige Darmbewegungen veranlasste, so ist bei Hungerkaninchen ein Ueberwiegen des Hemmungstonus anzunehmen.

Aus den Untersuchungen Swirski's wissen wir, dass das ohne Maulkorb hungernde Kaninchen bis zum achten Tage ungefähr einen ganz regelmässigen Kothkreislauf unterhält, eine vollkommene Unthätigkeit der Gedärme liegt also nicht vor. Solange das Thier sich auf den Füssen bält und noch nicht auf der Seite liegt, also seinen Koth noch erfassen kann, besteht noch immer eine Peristaltik. In solchen Fällen verhält sich der Mageninhalt zu dem des Blinddarmes wie $1: 4$. Da eine normale Füllung des Magens nur dann erreicht ist, wenn die Füllung desselben ungefähr der des Blind-

1) Schmiedeberg, Grundriss der Pharmakol. 4. Aufl. S. 109. Leipzig 1902. 
darmes gleichkommt, so müssen wir annehmen, dass das Gleichgewicht zwischen den beiden grössten Reservoirs des Nahrungsschlauches beim Hunger ohne Maulkorb gestört ist. Der Blinddarm erhält die constante Füllung mit ca. $14 \mathrm{~g}$ im Durchschnitte mit grosser Hartnäckigkeit, entspricht also der Füllung des Hungers von $1-2 \times 24$ Stunden. Es scheint dem hungernden Kaninchen eben mehr auf die Füllung des Blinddarmes, als die des Magens anzukommen. Man brauchte ja nur sich einen Nachlass der Hemmungswirkung zu denken, und der Magen könnte in seiner Füllung dem des Blinddarmes gleichkommen. Es bleibt aber eine Hemmungswirkung bestehen, wie es die Untersuchungen von Jacobj und v. Vámossy zeigen. Wenn nun ein ohne Maulkorb hungerndes Kaninchen Morphin erhält, so bildet sich, wie wir sahen, eine Vertheilung des Magendarminhaltes heraus, wie sie bei den normalen Kaninchen sich vorfindet (s. Tabelle III). Dass der Magen hierbei seinen vollen Inhalt abgegeben hat, haben wir oben gesehen; es ist also nichts in ihm liegen geblieben, was zu Beginn des Versuches in ihm vorhanden war, er ist jetzt mit Inhalt gefült, der verzehrten Koth darstellt. Der Blinddarm aber ist nicht so gefült wie bei den ohne Morphin hungernden, er enthält nur $10 \mathrm{~g}$, statt $14 \mathrm{~g}$ im Durchschnitte; zu Gunsten des Magens hat er von seinem Inhalte abgegeben. Die Hemmungswirkung des Splanchnicus ist also hier überwunden worden. Aus dem Versuche XIX von Jacobj ${ }^{1}$ ) geht hervor, dass nach $0,08 \mathrm{~g}$ Morphin. hydrochlor. die Hemmungswirkung des Splanchnicus abgeschwächt wurde. Da nun nach Entfernung der Nebennieren die Vagusreizung eine noch stärkere Bewegung der Därme : hervorrief, ist anzunehmen, dass es sich hierbei nicht um Veränderungen an den Darmganglien handelt, sondern um Abschwächung von Hemmungsimpulsen, die durch die Nebennieren vermittelt werden und wohl vom Rückenmarke herkommen. Es ist also eine Entleerung des Blinddarmes erreicht worden, wie sie beim ohne Morphin hungernden Kaninchen nicht stattfindet. Dadurch ist aber auch das Gleichgewicht zwischen Magen- und Blinddarminhalt hergestellt.

Während bei den Kaninchen, die ohne Maulkorb hungern, im Laufe der Hungerzeit nur unbedeutende Kothmengen, im Durchschnitt $1,7 \mathrm{~g}$, ausgeschieden werden, tritt bei den mit Maulkorb

1) Jacobj, l. c. S. 210 . 
bungernden meistens eine die Norm übersteigende Kothentleerung im Laufe der ersten zwei Tage ein. Ein durchfallartiger Charakter der Ausleerungen hängt von der Fütterungsweise der Thiere ab, indem Kartoffeln, als vorwiegende Nahrung, ganz besonders massige Fäces herbeiführen, während bei Haferfütterung die Kothmengen in der genannten Zeit wohl auch erhöht sind, aber kein Durchfall eintritt.

Die Ausleerungen betragen im Durchschnitte $24,3 \mathrm{~g}$ bei Kaninchen, die Kleeheu, Hafer und Brot erhalten. Indem sich Magen und Blinddarm entleeren, stellt sich in Bezug auf den Inhalt ein Gewichtsverhältniss wie $1: 13$ her. Auf den ersten Blick hin könnte es erscheinen, als wenn es sich hier um eine noch stärkere Splanchnicuswirkung handeln müsste als bei den ohne Maulkorb hungernden Kaninchen. Es ist das aber nicht der Fall; da der Magen sich bis auf ein Minimum, $0,3 \mathrm{~g}$, entleert, und im Blinddarme 3,7 als mechanisch nicht entfernbarer Koth nachbleiben, hat der Magendarmcanal das Minimum der Entleerung erreicht, dessen er überhaupt fähig ist. Da die Ausleerungen unentwegt vor sich gegangen sind, ist ein Ueberwiegen der motorischen Vagusimpulse vor der Hemmungswirkung des Splanchnicus anzunehmen. Es ist eine Regulirung in der Weise, dass vom Gehirne aus auf das Splanchnicuscentrum gerichtete Erregungen die Ausleerungen im Interesse des Organismus herabsetzen, wie man eigentlich hätte erwarten können, nicht erfolgt. Nach noch nicht veröffentlichten Untersuchungen von S wirski liess sich im Kochsalzbade an Maulkorbkaninchen kein Ueberwiegen der Splanchnicus- vor der Vaguswirkung nachweisen, wie das bei Kaninchen, die ohne Maulkorb hungerten, der Fall war.

Wenn nun bei dem Maulkorbhunger Morphineinspritzungen gemacht oder das Alkoloid per os eingeführt wird, so sieht man besonders bei den Thieren, die mit kleineren Morphingaben behandelt wurden, eine Vertheilung des Magendarminhaltes sich ausbilden wie bei normalen Thieren. Um dies zu erreichen, musste vor allen Dingen die Peristaltik des Magens herabgesetzt sein, denn sonst hätte nichts in ihm nachbleiben können, und andererseits durfte die Peristaltik im Blinddarme nicht zu sehr abgeschwächt werden, damit nicht eine Ueberfüllung desselben einträte. Das letztere scheint bei den mit grösseren Gaben Morphin, $0,65-0,76 \mathrm{~g}$, behandelten der Fall gewesen zu sein. Der Mageninhalt verhielt sich zu dem des Blinddarmes wie $1: 2,4$, während das Verhältniss bei den mit kleineren Dosen behandelten wie $1: 1,3$ war. Um der Frage näher zu gehen, 
ob wir es mit einer Wirkung des Morphins auf die Darmganglien oder mit einer durch das Alkaloid bewirkten Alteration der vom Centralnervensystem herkommenden Impulse zu thun haben, müssen wir auf die Verhältnisse beim gefütterten Kaninchen zurückgreifen. Wir sahen, dass eine Verminderung der Kothentleerung bei dem mit Hafer gefütterten Kaninchen nicht eintrat, trotzdem das Morphin. hydrochlor. in Dosen von $0,06 \mathrm{~g}$ in den ersten direi Versuchstagen, in den letzten drei jedoch zu 0,12 $\mathrm{g}$ täglich ein Mal per os eingeführt wurde. In der Vorperiode ohne Morphin wurde täglich durchschnittlich $9,4 \mathrm{~g}$ Koth entleert, während in der Morphinperiode $10,9 \mathrm{~g}$ ausgeschieden wurden. Application eines Maulkorbes bewirkte eine die Norm etwas übersteigende Ausleerung ohne Durchfall, wie das gewöhnlich bei Haferfütterung der Fall ist. Wir konnten also nichts finden, was auf eine Behinderung der Kothentleerungen, eine herabgesetzte Peristaltik hinwies. Es durfte erwartet werden, dass durch die locale Application eine directe Einwirkung des Morphins auf die Nerven, welche Reize aus dem Darmlumen empfangen und auf die motorischen Darmganglien übertragen, erzielt werde. Wäre eine Abstumpfung der genannten Nerven eingetreten, so hätte eine merkbare Verminderung der Kothentleerung die unausbleibliche Folge sein müssen. Nehmen wir andererseits an, dass die lähmende Wirkung des Morphin die vom Vaguscentrum ausgehenden Impulse abschwächt und die vom Splanchnicuscentrum ausgehenden Hemmungsimpulse herabsetzt, so könnte man sich vorstellen, dass trot\% einer centralen Beeinflussung die Peristaltik nicht gestört wird. Die abgeschwächten Vagusimpulse haben auch weniger Hemmungen zu überwinden. Die Verhältnisse beim Kaninchen sind derart, dass durch die beständige Sorge um die Aufrechterhaltung des Gleichgewichtes zwischen Magen und Blinddarm eine fast fortwährende Nahrungsaufnahme stattfindet, was bei Fleischfressern nicht der Fall ist. In dem gefülten Magen des Kaninchens vertheilt sich die eingeführte Morphinmenge auf die in ihm befiudliche Masse, und sehr bald gelangt das nicht resorbirte Morphin in den Dünndarm, der schnell passirt wird, um im Blinddarme, der eine dem Magen gleichkommende Masse Inhalt hat, sich zu vertheilen. Es kommt so das Morphin bei der steten Erneuerung des Inhaltes und der verhältnissmässig schnellen Passage desselben nicht zur Wirkung. Von dem in den Magen ausgeschiedenen Morphin sind gewiss Wirkungen zu erwarten, sie scheinen aber jedenfalls keinen die Peristaltik herabsetzenden Effect zu haben. 
Anders verhält es sich mit den Kaninchen, die während des Hungerns mit Morphin behandelt werden. Wir sahen, dass beim Hunger ohne Maulkorb durch Morphin eine Abschwächung der Hemmungswirkung des Splanchnicus eintrat, wodurch es dem Thiere möglich wurde, durch gefressenen Koth ein Gleichgewicht zwischen dem Magen- und Blinddarminhalte herzustellen. Beim Hunger mit Maulkorb tritt unter gleichzeitiger Morphinbehandlung die Entleerung res Magens bis zu einer Grenze ein, die nicht in merkbarer $\mathbf{A b}$ hängigkeit steht von der verabreichten Morphinmenge, mochte dieselbe subcutan oder per os verabfolgt sein. Wohl aber lässt sich eine Abhängigkeit der Füllung des Blinddarmes von der des Magens bei Dosen von $0,14-0,27 \mathrm{~g}$ constatiren: entweder entspricht die Füllung des ersteren der des letzteren vollkommen oder übersteigt sie um etwas. Während also die Magenfüllung eine für das Thier individuelle minimale Grenze erreicht, gibt dementsprechend der Blinddarm von seinem Inhalte ab. Wenn bei der Herabsetzung der Magenperistaltik das in den Magen ausgeschiedene Morphin auch eine Rolle spielt, so dürfte dieselbe doch nicht entscheidend sein. Fs ist vielmehr anzunehmen, dass das genannte Alkaloid eine Abschwächung der Impulse, die auf den Vagis zum Darmeanale gehen, bewirkt. Damit nun die in den Blinddarm gelangenden Darmcontenta nicht stauen, denn eine Abschwächung der Vagusimpulse würde den Splanchnicustonus vergrössern, tritt bier eine theilweise Lähmung des Centrums ein, was eine Entfernung des Ueberschüssigen zur Folge hat. Bei den mit grossen Morphinmengen, 0,66-0,76 g, behandelten Maulkorbkaninchen ist die excessive Füllung des Blinddarmes durch eine locale Beeinflussung der Darmganglien vermittelst des in diesen Fällen bedeutenden, in den genannten Darmtheil oder in den Magen ausgeschiedenen und hierher gelangten Morphinquantitäten zu erklären.

Aus der vorliegenden Arbeit glauben wir folgende Schlüsse ziehen zu dürfen:

1. Die Vertheilung des Magendarminhaltes normaler Kaninchen in der Weise, dass der Magen- und Blinddarminhalt in einem Gleichgewichte sich befinden, deutet darauf hin, dass diese Einrichtung für die Erhaltung des Organismus nothwendig ist.

2. Beim Hunger mit und obne Maulkorb tritt eine Störung dieses Gleichgewichtes ein, indem der Blinddarm dadurch das Uebergewicht erhält, dass der Magen sich mehr oder weniger entleert. 
3. Während des Hungers ohne Maulkorb subcutan einverleibtes Morphin. hydrochlor. führt durch eine Abschwächung des Splanchnicustonus eine Peristaltik herbei, die es dem Thiere ermöglicht, durch den verzehrten Koth eine Magendarmfüllung in dem Verhältnisse zu erzielen, wie sie den normalen Kaninchen zukommt.

4. Bei Maulkorbkaninchen bewirkt Morphin. hydrochlor. in Gaben von $0,14-0,27 \mathrm{~g}$ im Verlaufe von $3-5 \times 24$ Stunden einverleibt, durch eine Abschwächung motorischer, längs den Vagis zum Darmcanale gelangender Impulse, zum geringeren Theile durch locale Betäubung der Darmganglien, sowie durch eine theilweise Lähmung des Splanchnicuscentrums eine Vertheilung des Magendarminhaltes, wie sie derjenigen normaler Kaninchen nahe kommt. Dosen von $0,66-0,76 \mathrm{~g}$ Morphin hydrochlor. bringen ein Ueberwiegen des Blinddarminhaltes wahrscheinlich dadurch zu Stande, dass eine locale Beeinflussung der Blinddarmganglien durch in den Darm ausgeschiedenes Morphin eintritt.

5. Während der Morphinbehandlung nimmt der Magendarminhalt beim Hunger mit und ohne Maulkorb merklich an Gewicht zu.

Herrn Privatdocenten der kaiserlichen Universität zu Jurjew (Dorpat) Dr. med. G. Swirski bitte ich, meinen aufrichtigsten Dank entgegen $\mathrm{zu}$ nehmen für die freundliche Ueberlassung des Themas und eines Theiles des Materials der Arbeit wie auch für die liebenswürdige Unterstützung mit Rath und That bei der Abfassung dieser Arbeit. 